Musées, Patrimoine et Culture scientifiques et techniques

$188 \mid 2020$

mars-avril 2020

\title{
Citamix : marathon créatif pour outils de médiation innovants
}

Héloïse Patela, heloise.patela@citadelle.besancon.fr et Gaëlle Cavalli

\section{OpenEdition}

\section{Journals}

Édition électronique

URL : https://journals.openedition.org/ocim/3636

DOI : 10.4000/ocim.3636

ISSN : 2108-646X

Éditeur

OCIM

Édition imprimée

Date de publication : 1 mars 2020

Pagination : 22-29

ISSN : 0994-1908

Référence électronique

Héloïse Patela, heloise.patela@citadelle.besancon.fr et Gaëlle Cavalli, «Citamix : marathon créatif pour outils de médiation innovants », La Lettre de I'OCIM [En ligne], 188 | 2020, mis en ligne le 01 mars 2021, consulté le 17 juillet 2021. URL : http://journals.openedition.org/ocim/3636 ; DOI : https://doi.org/ 10.4000/ocim.3636

Ce document a été généré automatiquement le 17 juillet 2021.

Tous droits réservés 


\section{Citamix : marathon créatif pour outils de médiation innovants}

Héloïse Patela, heloise.patela@citadelle.besancon.fr et Gaëlle Cavalli

Illustration d'après le visuel de Citamix apposé sur une vuede la Citadelle de Besançon.

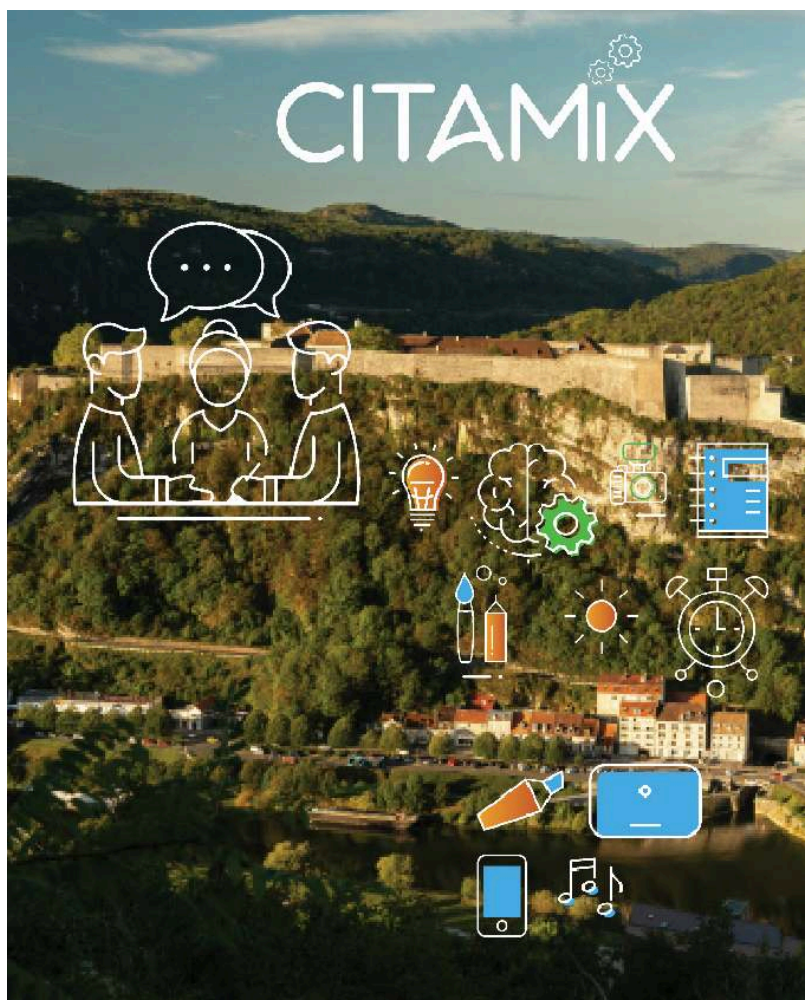

(c) photographie :Christophe Roy ;éléments graphiques : Citadelle de Besançon

1 À travers l'exploration de nouvelles méthodes de travail collaboratives, les organisateurs de Citamix ont souhaité connaître la perception que peuvent avoir de 
jeunes étudiants d'un patrimoine architectural majeur, comme la Citadelle de Besançon.

Citamix est l'aboutissement de plusieurs années de réflexion autour de la mise en place d'un projet collaboratif et coopératif à la Citadelle de Besançon. En reprenant les valeurs clés des Museomix ${ }^{1}$ (ouverture, expérimentation et partage), le service Valorisation du monument Vauban s'est donné pour mission de réunir des étudiants de l'université de Franche-Comté afin qu'ils réfléchissent et prototypent des outils de médiation innovants pour le monument.

3 La Citadelle de Besançon a souhaité s'appuyer en priorité sur les ressources et richesses humaines du territoire pour sa première édition, contrairement aux Museomix qui sollicitent des talents issus de toute la France. Les équipes, composées de six étudiants de diverses filières, avaient trois jours et une nuit pour créer un outil qui réponde à la problématique : "Jouez avec le monument Vauban!».

Depuis 2008, la Citadelle et les fortifications de la ville de Besançon sont inscrites sur la liste du Patrimoine mondial de l'Unesco conjointement avec onze sites majeurs de l'œuvre de l'ingénieur Sébastien Le Prestre de Vauban. Ce site d'exception comprend aujourd'hui trois musées de France (muséum d'Histoire naturelle, musée Comtois et musée de la Résistance et de la Déportation) ainsi qu'un parc zoologique et accueille une moyenne de 270000 visiteurs par an.

5 Les projets de médiation issus de l'événement ont été créés exclusivement pour le monument. Même si le projet original ne s'adressait pas à l'ensemble des pôles de la Citadelle, il peut néanmoins facilement être décliné dans les différents musées du site ou dans d'autres structures culturelles.

Phase de conception des outils de médiation imaginés.

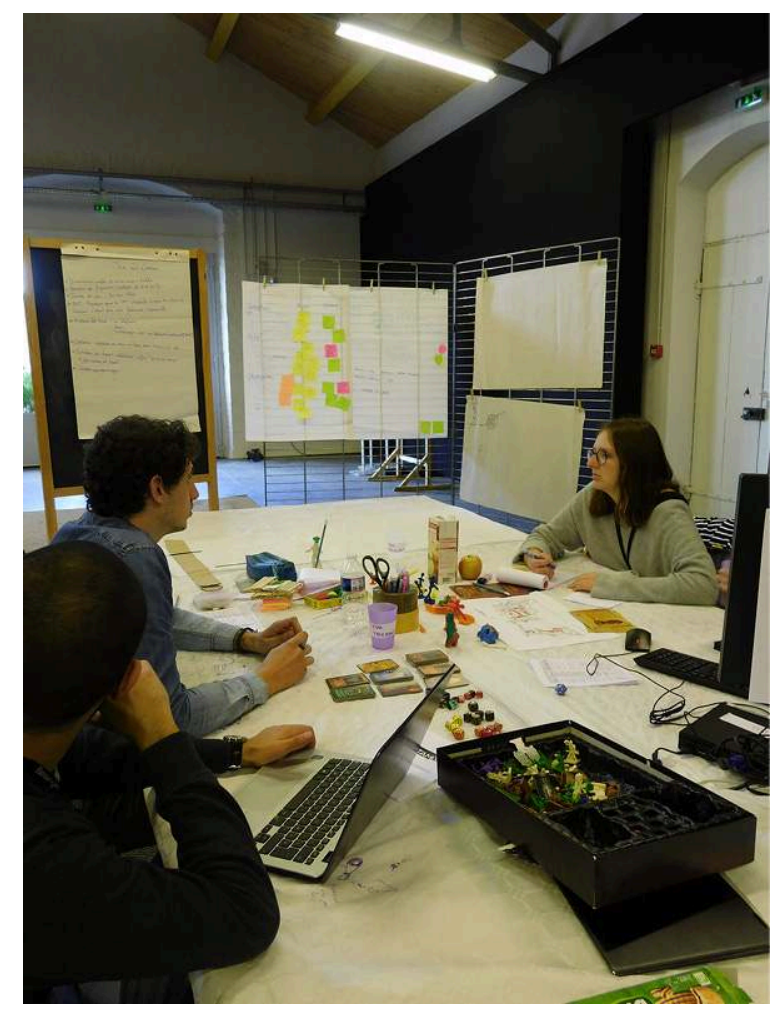

(c) Hermine Chapron 


\section{S'inscrire dans de nouvelles tendances de médiation}

6 $12 \%$ d'étudiants. La question de savoir si les outils de médiation actuellement disponibles sur le site sont adaptés aux attentes de ce public nous paraissait donc très pertinente. La proposition de la Citadelle visait également à la construction d'un lien privilégié avec le public étudiant, en lui donnant l'opportunité d'être acteur de la valorisation de ce patrimoine pour mieux se l'approprier. Mettre en place ce type d'action collaborative représente aussi pour le site une façon de s'inscrire dans les nouvelles tendances de la médiation et de renouveler l'image de la Citadelle.

Qu'entendons-nous par nouvelles tendances de médiation ? L'outil de médiation joue le rôle d'intermédiaire entre un public et une œuvre/exposition/monument. Il doit dès lors être adapté aux attentes et modes de décryptage et de compréhension du visiteur. Ceci implique une adaptation constante en fonction des changements sociétaux majeurs (développement du numérique, nouveaux modes de consommation de biens culturels...). Ainsi, 78 \% des internautes âgés de 15 ans et plus ont consommé en 2018 des biens et services culturels en ligne en France ${ }^{3}$. On peut donc en déduire qu'un visiteur aujourd'hui ne possède pas les mêmes clés de lecture ni la même méthode d'exploration et de découverte qu'un visiteur d'il y a 15 ans. Si les institutions culturelles veulent attirer des visiteurs, elles doivent évoluer au même rythme que la société.

Les institutions culturelles développent de nouvelles manières de visiter : possibilité de dormir au musée (Plan B du Mucem) ${ }^{4}$, apparition d'activités ludiques et participatives telles que les escape games (comme Inside Opéra à l'Opéra Garnier) ${ }^{5}$. Ces expériences de visite peuvent parfois même rentrer dans le cadre de l'insolite comme ce fut le cas du musée Léopold à Vienne où en février 2013 le public fut invité à se déshabiller lors de l'exploration d'une exposition autour de la nudité masculine. Ceci s'ajoute à la multiplication des expositions temporaires et des événementiels, à la volonté de confrontation à l'art contemporain, à la théâtralisation des visites, à l'augmentation des nocturnes et au développement des applications et supports numériques.

9

Selon l'étude menée par le $\mathrm{Credoc}^{6}, 81 \%$ des français pensent que pour rendre les musées accessibles à un public plus jeune, une des mesures prioritaires à instaurer serait d'associer davantage ce public à la vie du musée ${ }^{7}$. Le rapport de la mission Musées $d u X X I^{e}$ siècle souligne également l'importance de l'émergence de tiers-lieux dans les structures culturelles qui accompagnent l'apparition accrue d'actions collaboratives dans la société. En effet, des projets où la collaboration joue un rôle central permettent au public d'investir les espaces culturels différemment et ainsi de diminuer certaines barrières qui peuvent freiner leur fréquentation. Parmi ces projets collaboratifs on retrouve le Biblio Remix, un événement du type hackathon autour des services de bibliothèque. Ce projet vise à réunir des individus aux compétences variées afin qu'ils construisent ensemble leur bibliothèque idéale. Plusieurs éditions ont eu lieu dans différentes villes ${ }^{8}$. 
Les impressions photographiques équipés de puces NFC développées par l'équipe Com'Gang pour reproduire la cour de la Citadelle, fermée au public.

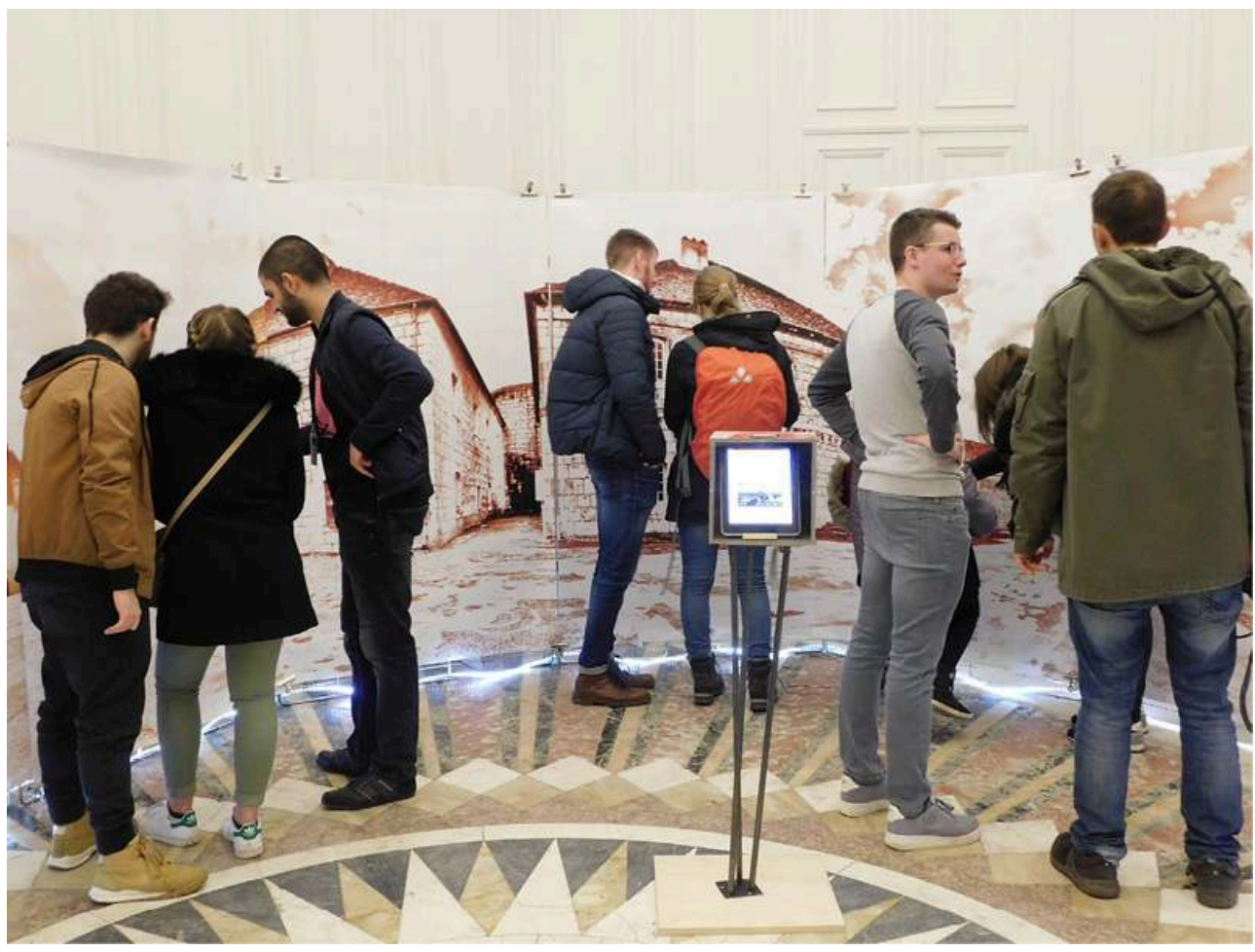

(c) Hermine Chapron

Citamix s'inscrit dans cette tendance et permet notamment de mieux comprendre l'évolution des attentes et centres d'intérêt d'un public jeune. Ce marathon, où la créativité joue un rôle clé, représente une expérience singulière durant laquelle les participants ont une occasion d'appliquer les connaissances acquises lors de leurs cursus, d'ajouter une expérience originale à leur $\mathrm{CV}$ tout en découvrant différents métiers (médiateur du patrimoine, développeur, web designer, concepteur de jeux et d'escape games...).

11 Pour l'université de Franche-Comté, co-organisatrice de l'événement, ce fut l'occasion de s'inscrire dans une démarche coopérative avec une structure touristique et culturelle. Citamix s'inscrivait parfaitement dans un objectif crucial pour l'université à l'heure actuelle : développer l'ouverture culturelle des étudiants.

12 L'événement n'aurait pu voir le jour sans la participation enthousiaste de divers professionnels issus de corps de métiers tout aussi intéressants que différents, qui ont joué le rôle de personnes ressources : conseils aux étudiants, explication du fonctionnement des machines et leurs contraintes, transmission de connaissances historiques, comme par exemple via la modélisations de personnages du XVII ${ }^{\mathrm{e}}$ siècle ou via des plans anciens de la Citadelle et des dessins évoquant la vie quotidienne, formation rapide au montage de petits films, test des règles du jeu et même création d'une plateforme de développement d'application. Nous avons ainsi pu compter sur l'appui de l'association Collectif Ludique Bisontin (organisateurs du festival Ludinam dédié aux jeux à Besançon), du Fablab des Fabriques (service municipal), et des sociétés Livdéo (création de plateformes numériques), Héritage Virtuel (technologie et modélisation 3D) et La Clé du Bastion (créateurs d'escape games). Le projet a par ailleurs 
bénéficié du soutien financier de la Direction régionale des affaires culturelles Bourgogne-Franche-Comté, de la Région Bourgogne-Franche-Comté et du Département du Doubs.

Le plateau de jeu créé par l'équipe TNT reproduit le siège de Besançon de 1674.

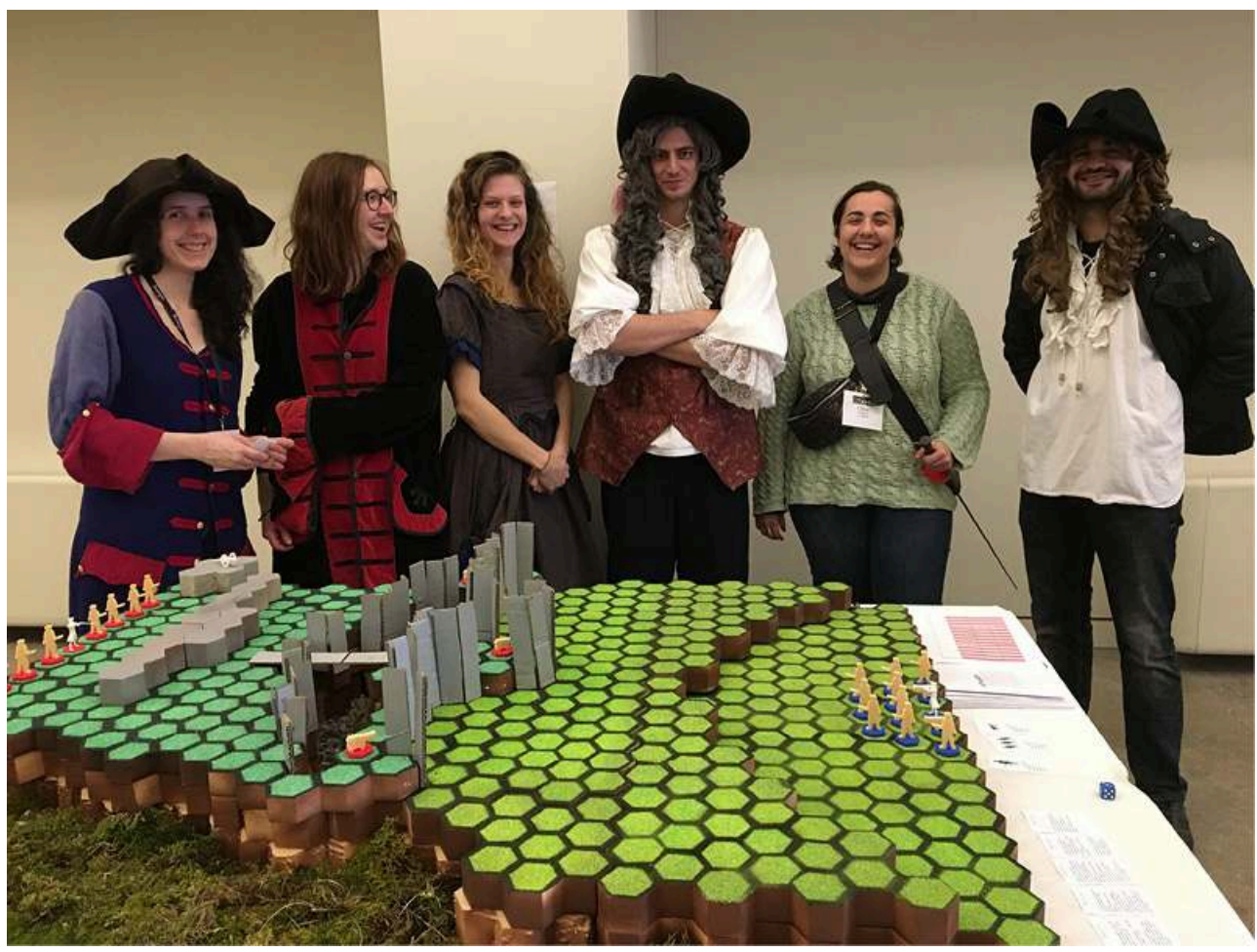

(c) Hermine Chapron

\section{L'évènement en quelques mots}

Citamix est un moment de rencontre entre des étudiants aux compétences variées et des professionnels de différents secteurs. L'événement s'est déroulé du jeudi 7 au dimanche 10 mars 2019. Les dates ont été choisies en fonction du calendrier universitaire afin de ne pas interférer avec les cours, examens, stages ou autres contraintes qui auraient pu se présenter pour les étudiants. La participation était entièrement gratuite et tous les repas ont été fournis par la Citadelle.

Les organisateurs de l'événement s'étaient rendus directement à la rencontre des étudiants et de certains responsables de filières à partir du mois de janvier. Suite à notre campagne de recrutement, 21 jeunes ont souhaité faire partie de l'aventure Citamix. Plusieurs filières étaient représentées, dont certaines non directement en lien avec les problématiques historiques, culturelles ou patrimoniales, ce qui faisait la richesse de cette édition et de la rencontre entre ces jeunes : InformationCommunication, Métiers de l'exposition et Technologies de l'information, Archéologie, Histoire de l'art, Ingénierie pédagogique et Histoire, Civilisation et Patrimoine, Pharmacie, Droit.

15 Les inscrits ont pu prendre part à une visite privilégiée avec la médiatrice du monument en amont de l'événement. L'objectif de cette rencontre était de les 
familiariser avec l'histoire et l'architecture du lieu et de leur montrer les dispositifs de médiation déjà mis en place (application numérique, maquette, spectacle multimédia...). Il était essentiel qu'ils puissent s'approprier le patrimoine sur lequel ils allaient travailler avant de se lancer dans la construction d'un dispositif de valorisation de celui-ci. Cette phase de découverte était accompagnée d'une présentation sur les étapes à suivre pour mener un projet sur un temps limité afin que les étudiants impliqués aient quelques orientations avant de se lancer dans la conception de leur prototype. Cette sensibilisation à la gestion de projet avait pour but de leur faire gagner du temps et de leur montrer comment appréhender les enjeux de ce type de démarche.

\section{Trois jours et une nuit pour concevoir et construire}

16 L'événement a débuté le jeudi soir à 18 heures par un apéritif dinatoire où les personnes ressources ont pu présenter leurs compétences respectives et les participants ont eu le temps d'échanger avant de se lancer dans la constitution de leurs équipes et la conception de leur projet. L'idée était d'avoir des équipes constituées de personnes issues de différentes filières afin de favoriser le partage de compétences.

À partir de 21 heures, les groupes composés de 4 à 6 étudiants ont commencé à travailler. Munis de beaucoup d'imagination et de motivation, ils ont pu passer une nuit blanche à phosphorer. Pendant la phase de conception qui a duré jusqu'au vendredi après-midi, ils ont été quotidiennement filmés afin donner à voir l'avancement de leur projet et expliquer leur démarche9. Ensuite, ils ont présenté et expliqué leur projet auprès des partenaires. Filmés quotidiennement, un montage été réalisé pour donner à voir l'avancement de leur projet et expliquer leur démarche. Dès le samedi matin, la phase de prototypage débutait : cartons, bois, peinture, puces $\mathrm{NFC}^{10}$, Arduino ${ }^{11}$, découpe laser, imprimantes 3D étaient quelques-uns des outils mis à disposition des équipes. 


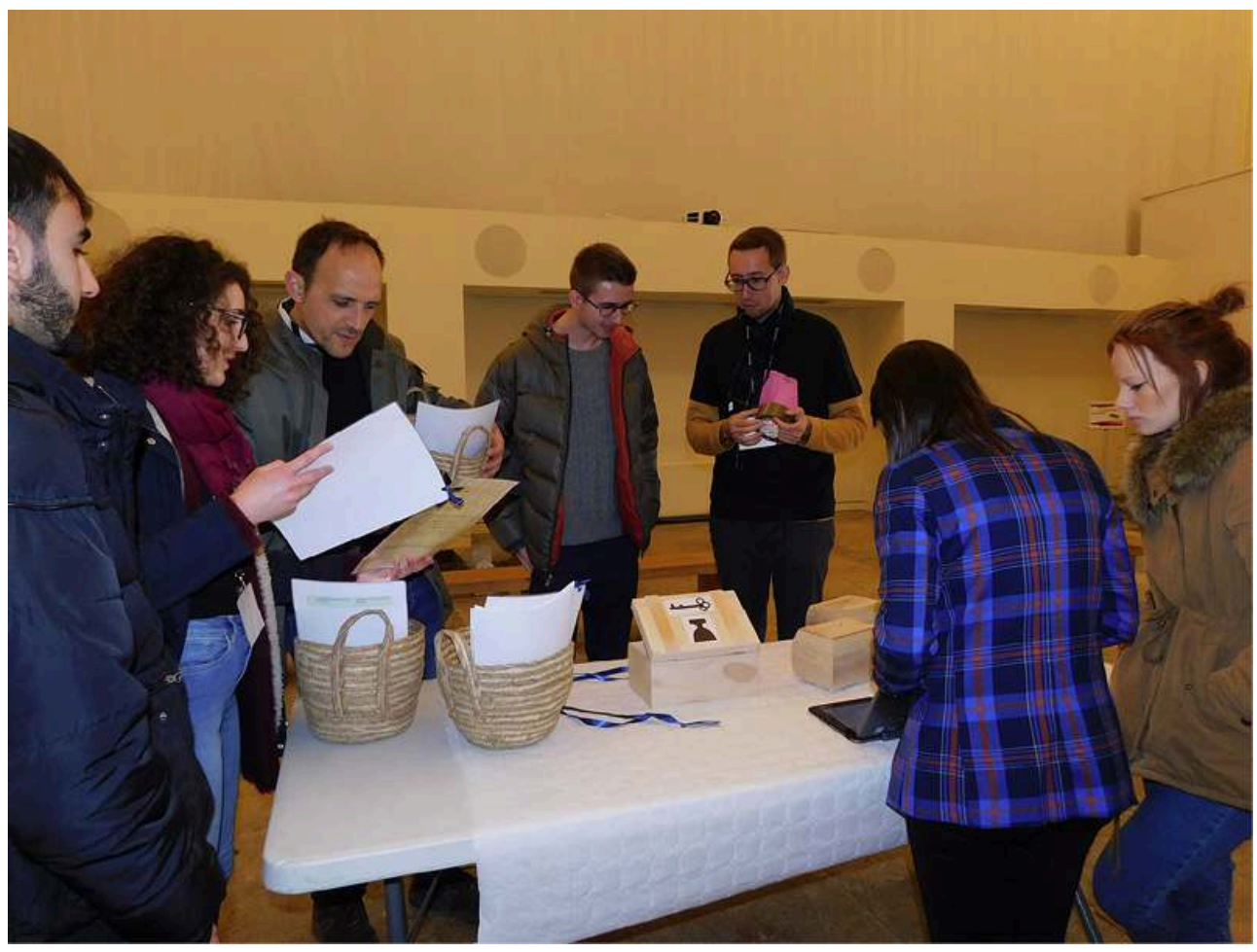

(C) Hermine Chapron

Une problématique à laquelle les équipes ont dû rapidement répondre était celle des éléments caractéristiques d'une médiation innovante. Pour les organisateurs, un outil de médiation innovant ne se limite pas à un outil numérique. Il doit favoriser l'appropriation du patrimoine, prendre en considération les visiteurs et leurs modes de visites, dégager quelque chose au-delà de la visite effective (émotion, souvenir, connaissance nouvelle, compréhension d'un sujet particulier) et permettre d'établir un lien entre l'individu et l'œuvre/exposition/monument. Pour répondre à cet objectif, il faut avant tout définir quel rapport au monument le visiteur aura grâce à l'outil. Pour ne pas brider la créativité des participants, les organisateurs avaient pris le parti de ne pas définir à l'avance certains éléments tels que le public cible, le lieu d'implantation de l'outil ou encore la forme que devait prendre le dispositif (maquette, application numérique, borne musicale...).

Après cette période de marathon créatif, les outils de médiation conçus ont pu être présentés au public de la Citadelle, le dimanche 10 mars. Cette présentation répondait à une double volonté des organisateurs : celle de valoriser le travail des étudiants et celle de faire vivre une expérience inédite aux visiteurs de la Citadelle. Pour continuer dans cette démarche d'expérience participative, il était important de partager le vécu des équipes sur les réseaux sociaux de la Citadelle (Twitter, Facebook et Instagram). Le but étant aussi que les internautes puissent interagir et voter pour leur prototype « coup de cœur » sur les réseaux sociaux. L'évènement a également été beaucoup relayé depuis les comptes personnels des différents participants. 
Test de l'application numérique Vauban a perdu la mémoire imaginée par l'équipe Scéna Cita.

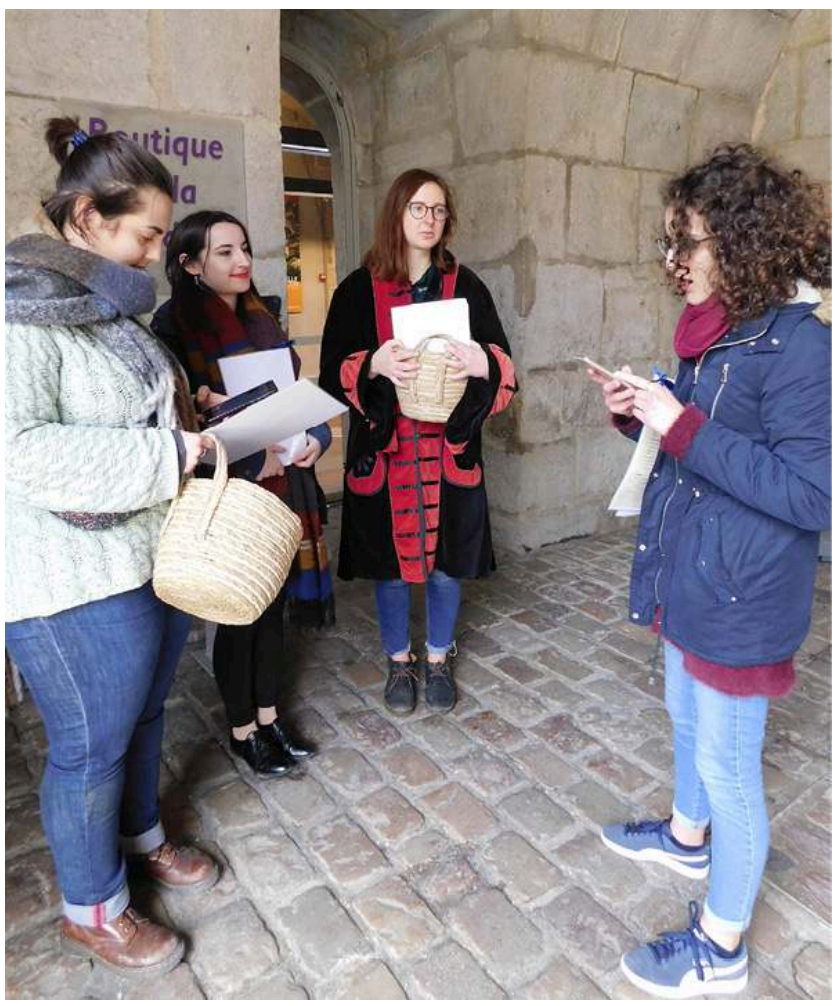

(c) Hermine Chapron

\section{Les projets : trois jeux et une maquette connectée}

\section{captivants que ludiques. Les équipes ont su répondre au défi de " jouer avec le} monument Vauban » avec brio. Chaque projet permettait aux visiteurs d'être plus actifs dans leur visite en jouant, interprétant ou testant de nouveaux outils. Il ne faut pas non plus négliger le rôle des " personnes ressources " qui ont su, tout au long de la manifestation, orienter et accompagner les équipes dans la mise en place de leur idée.

- L'équipe TNT a créé un jeu de plateau qui mêle stratégie et hasard. Les visiteurs, dans un premier temps, sont invités à connaître la Citadelle à travers un livret comprenant des questions sur le site (notamment la position géostratégique et l'adaptation au terrain). Les joueurs sont ensuite invités à effectuer une partie du jeu de plateau. Ils se retrouvent soit dans la position d'attaquant, soit dans la position de défenseur. Deniz, Lucas, Eva, Laura, Chloé et Juliette ont ainsi construit un plateau de jeu représentant la Citadelle, créé les pions en polystyrène et inventé toutes les règles de jeu.

- Les membres de l'équipe Cita Dream (Cécile, Julie, Baptiste, Pierrick et Élise) se sont donnés pour mission de mettre en valeur le patrimoine à travers un jeu de piste sous forme d'application numérique. À travers la résolution d'énigmes qui amènent le visiteur à découvrir différents lieux clés de la Citadelle, les joueurs doivent aider le fantôme de Vauban à retrouver la mémoire. Une fois les différents défis relevés, le personnage de Vauban explique aux visiteurs comment il a conçu la Citadelle. Une 
manière originale d'expliquer les particularités architecturales qui font de la Citadelle de Besançon une forteresse unique en France.

- Le troisième groupe Scéna Cita a, quant à lui, décidé de mettre en place une animation adaptée à tous les publics. Les visiteurs sont invités à suivre un itinéraire en partant à la recherche de $Q R$ codes dispersés sur le site. Une fois chaque $Q R$ code scanné, celui-ci renvoie à la vidéo d'un personnage de la Citadelle (aumônier, boulanger...). À la fin du visionnage des vidéos, une énigme permet de passer à l'étape suivante. À travers le parcours, les joueurs trouvent des boîtes à indices. Chloé, Claire, Éléa et Adam ont créé deux scénarios afin que deux équipes puissent suivre l'itinéraire simultanément et être en compétition.

24 - Les membres de la Com'Gang (Luca, Pauline, Gabriel, Corentin, Léo et Valentin) ont souhaité combiner les mondes physique et numérique. Ces étudiants sont partis du constat que beaucoup d'espaces de la Citadelle (notamment les bâtiments administratifs) n'étaient pas ouverts au public. Pour pallier ce problème, ils ont recréé la cour principale de la Citadelle à l'aide de supports habillés avec des impressions photographiques derrière lesquelles ils ont installé des puces NFC. En passant un téléphone ou une tablette compatible sur la surface située devant ces puces, des contenus révélant la fonction originelle des bâtiments s'affichaient.

La thématique de cette première édition, Jouez avec le monument Vauban, explique la prépondérance du ludique parmi les dispositifs créés. Cependant, chaque projet avait une vocation didactique, ce qui est le propre des outils de médiation : le projet de jeu de piste avec vidéos permettait de découvrir la diversité des métiers présents à la Citadelle à l'époque de Vauban (aumônier, chirurgien...) Le projet d'exposition digitale permettait au-delà des murs des bâtiments pris en photo de comprendre l'ancienne fonction des bâtiments aujourd'hui dévolus aux musées et à l'administration, l'application numérique Vauban a perdu la mémoire permettait quant à elle de comprendre l'œuvre architecturale, et le jeu de plateau reconstituait le siège de Besançon de 1674 , et dans sa première partie permettait d'appréhender le rôle géostratégique du site. Chacun a donc choisi un angle d'approche différent, ce qui était d'autant plus intéressant.

\section{Quelles perspectives pour Citamix ?}

S'il a demandé un investissement en temps non négligeable avec une personne à plein temps pendant une durée d'environ trois mois, le projet fut également très enrichissant du point de vue du personnel de la Citadelle. Outre l'implication du service Valorisation du monument, d'autres services ont pu être associés à la mise en place de la manifestation. Certains membres du service Accueil/Surveillance se sont investis dans le projet en proposant des idées pour l'aménagement du lieu ou des techniques pour permettre de terminer les outils et ont aussi contribué à l'organisation et au bon déroulement de Citamix. Les organisateurs ont pu changer leur quotidien pendant trois jours et explorer leur lieu de travail sous un autre angle. Ainsi la médiatrice du monument a pu sortir de son rôle habituel de présentation et création de contenus et être plus sur un poste d'accompagnement de projet et de personne ressource. L'événement a également suscité l'intérêt de plusieurs membres du personnel, qui sont venus à maintes reprises étudier le niveau d'avancement de chaque prototype. Citamix s'est donc avéré fédérateur pour les membres du personnel de la Citadelle. 
L'engouement du public est un autre point positif de l'événement. En effet, outre la centaine de visiteurs présents sur le site lors de la restitution, 2165 personnes se sont manifestées sur les réseaux sociaux afin de voter pour leur projet coup de cœur. Cet engouement démontre que ce type d'initiative peut favoriser l'adhésion d'un nouveau public. Transformer la Citadelle en un réel lieu d'échange et de vie est l'un des enjeux de ce type d'événement.

Une des questions sur lesquelles l'équipe de la Citadelle travaille actuellement est la manière d'exploiter les quatre créations des équipes, afin que le projet ne soit pas éphémère et que le public de la Citadelle puisse en profiter plus largement. Ainsi le plateau de jeu de l'équipe TNT a pu être présenté lors du festival Ludinam organisé par le Collectif ludique bisontin du 3 au 5 mai 2019. Une réflexion avec l'aide de certains participants est en cours pour définir des pistes possibles de pérennisation et d'exploitation de chaque outil.

L'événement a rempli un autre objectif primordial : celui de créer de réels liens entre les participants et les professionnels présents. Deux d'entre eux ont ainsi été recrutés par le service Valorisation du monument, l'un pour être animateur du nouvel escape game de la Citadelle et l'autre pour un contrat d'apprentissage d'un an. Les retours des étudiants sur leur expérience furent très positifs. Parmi ceux-ci, nous pouvons citer Adam : «c'était une super expérience et j'en garderai de très bons souvenirs "; Eva : "C'est très formateur "; Chloé : "C'est réjouissant de voir que nos idées se mettent en place, on est plus soudés après l'expérience, c'est enrichissant d'avoir travaillé tous ensemble » ou encore Léo : "On a le sentiment du devoir accompli, c'était parfait ».

$\mathrm{Vu}$ l'intérêt des projets qui ont vu le jour, l'enthousiasme des participants (étudiants et personnes ressources) et le bon retour du public, reconduire l'événement pour une deuxième édition apparaît comme une évidence. D'autres structures culturelles de la région travaillent également à la possibilité de réaliser prochainement des manifestations basées sur ce modèle - preuve que l'aspect collaboratif et coopératif a bien sa place dans les institutions culturelles -. Il est également envisagé de développer cette manifestation en réseau avec des sites susceptibles de présenter des problématiques complémentaires à celles de la Citadelle. D'autres fortifications sont envisagées pour le moment.

Est-ce que la représentation de la Citadelle mise en avant dans les quatre projets permettra réellement d'attirer un nouveau public et de donner une autre vie à cet ensemble architectural ? Tout porte à le croire. Les équipes ont créé des outils qu'elles aimeraient rencontrer aujourd'hui dans un site culturel et touristique et cette envie est sans doute partagée par d'autres personnes appartenant à cette même tranche d'âge.

\section{NOTES}

1. Museomix est un marathon annuel créatif international de 3 jours dans les musées. Consacré aux nouvelles formes de médiation et au numérique dans les musées, l'objectif de cet évènement est de concevoir et prototyper des expériences innovantes : www.museomix.org 
2. Selon une étude réalisée pendant la saison estivale de 2016 par Kheolia.

3. Ministère de la Culture, Département des études, de la prospective et des statistiques. Chiffres Clés 2018 - Statistiques de la culture et de la communication. Paris : Presses de Sciences Po, 2018, 288 p.

4. Le musée des civilisations de l'Europe et de la Méditerranée de Marseille propose un programme nocturne et invite les visiteurs à dormir dans le musée dans le cadre de Plan B : www.mucem.org/programme/planb-2019-dormir-au-musee-experiences-nocturnes-insolites

5. L'aventure immersive d'Inside Opéra propose au public de résoudre le mystère du Fantôme de l'Opéra : www.inside-infos.fr/opera

6. Bigot R., Hoibian S. et Müller J. Les visites du patrimoine culturel et la question de la gratuité, Collection des rapports Crédoc, $\mathrm{n}^{\circ} 326,2016$.

7. Eidelman J. (dir.), ministère de la Culture et de la Communication, Direction générale des Patrimoines. Inventer des musées pour demain, rapport de la mission Musées du XXIe siècle. Paris : La Documentation française, 2017, $251 \mathrm{p}$.

8. Biblio Remix est un dispositif d'expérimentation, d'invention et de création participatives, autour des services en bibliothèque : https://biblioremix.wordpress.com

9. Un court film disponible sur Youtube récapitule le déroulé de Citamix : bit.ly/video-citamix

10. Technologie qui permet l'échange d'informations sans contact entre deux appareils qui en sont équipés.

11. Carte électronique (en licence libre) sur laquelle se trouve un microcontrôleur destiné, notamment, à la programmation multimédia interactive, à construire des objets interactifs indépendants ou bien à être connecté à un ordinateur pour communiquer avec ses logiciels.

\section{RÉSUMÉS}

En mars 2019, la Citadelle de Besançon initiait Citamix, un projet collaboratif et coopératif impliquant des étudiants dans la création d'outils de médiation. Un an après cette première édition intitulée Jouez avec le monument Vauban, les auteurs reviennent sur leur démarche et les perspectives offertes par ce dispositif.

\section{INDEX}

Mots-clés : dispositif ludique, dispositif participatif, médiation, jardin zoologique

\section{AUTEURS}

\section{HÉLOÏSE PATELA}

Étudiante en Master II Management du Tourisme et de la Culture - IAE Dijon, en alternance dans le service Valorisation du Monument Vauban, Citadelle de Besançon-patrimoine mondial

\section{GAËLLE CAVALLI}

Responsable du service Valorisation du Monument Vauban, Citadelle de Besançon-patrimoine mondial 
gaelle.cavalli@citadelle.besancon.fr 